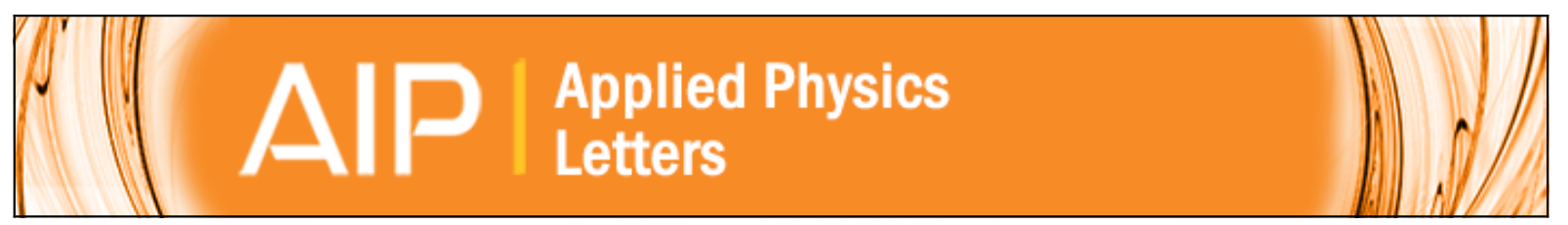

\title{
Optimizing the internal electric field distribution of alternating current driven organic light-emitting devices for a reduced operating voltage
}

Markus Fröbel, Simone Hofmann, Karl Leo, and Malte C. Gather

Citation: Applied Physics Letters 104, 071105 (2014); doi: 10.1063/1.4865928

View online: http://dx.doi.org/10.1063/1.4865928

View Table of Contents: http://scitation.aip.org/content/aip/journal/apl/104/7?ver=pdfcov

Published by the AIP Publishing

\section{Articles you may be interested in}

Fundamental properties of light-emitting liquid crystal cells operated under alternating voltage

J. Appl. Phys. 114, 053108 (2013); 10.1063/1.4817173

Degradation studies on high-voltage-driven organic light-emitting device using in situ on-operation method with scanning photoelectron microscopy

Appl. Phys. Lett. 93, 133310 (2008); 10.1063/1.2994668

Improving organic light-emitting devices by modifying indium tin oxide anode with an ultrathin tetrahedral amorphous carbon film

J. Appl. Phys. 98, 046107 (2005); 10.1063/1.2032610

Mixed host organic light-emitting devices with low driving voltage and long lifetime

Appl. Phys. Lett. 86, 103506 (2005); 10.1063/1.1879093

High-efficiency polymer light-emitting devices using organic salts: A multilayer structure to improve light-emitting electrochemical cells

Appl. Phys. Lett. 81, 214 (2002); 10.1063/1.1490635

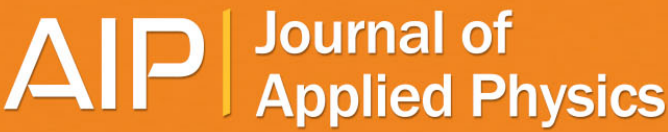

Journal of Applied Physics is pleased to announce André Anders as its new Editor-in-Chief 


\title{
Optimizing the internal electric field distribution of alternating current driven organic light-emitting devices for a reduced operating voltage
}

\author{
Markus Fröbel, ${ }^{1, a)}$ Simone Hofmann, ${ }^{1}$ Karl Leo, ${ }^{1}$ and Malte C. Gather ${ }^{1,2}$ \\ ${ }^{1}$ Institut für Angewandte Photophysik, Technische Universität Dresden, George-Bähr-Strasse 1, \\ 01069 Dresden, Germany \\ ${ }^{2}$ School of Physics and Astronomy, University of St Andrews, North Haugh, St Andrews KY16 9SS, \\ United Kingdom
}

(Received 7 November 2013; accepted 1 February 2014; published online 18 February 2014)

\begin{abstract}
The influence of the thickness of the insulating layer and the intrinsic organic layer on the driving voltage of $p-i-n$ based alternating current driven organic light-emitting devices (AC-OLEDs) is investigated. A three-capacitor model is employed to predict the basic behavior of the devices, and good agreement with the experimental values is found. The proposed charge regeneration mechanism based on Zener tunneling is studied in terms of field strength across the intrinsic organic layers. A remarkable consistency between the measured field strength at the onset point of light emission (3-3.1 MV/cm) and the theoretically predicted breakdown field strength of around $3 \mathrm{MV} / \mathrm{cm}$ is obtained. The latter value represents the field required for Zener tunneling in wide band gap organic materials according to Fowler-Nordheim theory. AC-OLEDs with optimized thickness of the insulating and intrinsic layers show a reduction in the driving voltage required to reach a luminance of $1000 \mathrm{~cd} / \mathrm{m}^{2}$ of up to $23 \%(8.9 \mathrm{~V})$ and a corresponding $20 \%$ increase in luminous efficacy. (C) 2014 AIP Publishing LLC. [http://dx.doi.org/10.1063/1.4865928]
\end{abstract}

Inorganic alternating current electroluminescent devices (AC-ELs) were first reported in 1936 and have been under investigation for the past seven decades. ${ }^{1}$ The key-advantage of these devices is their ruggedness and long-term reliability which is why they can often be found in industrial and medical equipment as well as in applications in the military sector. ${ }^{2}$ AC-ELs are based on a phosphor layer which is doped with luminescent impurities. The presence of a high field leads to impact excitation of these impurities and the subsequent decay to the ground state results in light emission. ${ }^{3}$ However, due to the low photoluminescent quantum yield of inorganic phosphors and due to the lack of full color capability - a problem which has only quite recently been solved-AC-ELs are nowadays mostly found in niche applications where their unique properties are required.

In contrast to inorganic phosphors, organic materials offer a number of advantages, in particular a higher efficiency, easier processibility, and a wide selection of emitter materials spanning the entire visible spectrum. Several efforts towards AC organic light-emitting devices (AC-OLEDs) have been made in the recent past, and even white-emitting AC-OLEDs have been successfully demonstrated. ${ }^{4-8}$ However, the majority of these devices rely on charge injection from one or even both electrodes and show poor performance when operated in a full insulating, capacitively coupled mode, i.e., in a configuration where two insulators prevent charge injection from both electrodes. For this particular configuration, the most promising approach in terms of brightness and efficiency is a $\mathrm{p}-\mathrm{i}-\mathrm{n}$ based architecture in which a single emissive unit is surrounded by doped hole and electron transport layers (HTL/ETL) and by a pair of insulating layers; the whole stack is then sandwiched between two electrodes. ${ }^{9-12}$ The insulating layers prevent current injection

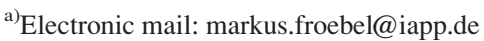

from the electrodes when a DC voltage is applied to the device. Application of a forward voltage pulse, however, drives free electrons and holes from the doped transport layers into the emissive unit, where they can radiatively recombine. The emission from the device stops once the free charge carriers available within the device are depleted. The device can be restored by applying a reverse voltage that replenishes the charge reservoir in each transport layer through a Zener tunneling process. ${ }^{12,13}$ The tunneling mechanism strongly depends on the electric field across the intrinsic layers within the $\mathrm{p}-\mathrm{i}-\mathrm{n}$ based AC-OLED. This field can be tuned by the capacitance of the insulating layers as well as by the thickness of the intrinsic organic layers.

In this Letter, we study the influence of the thickness of the insulator layer and the intrinsic organic layer on the driving voltage of $\mathrm{p}-\mathrm{i}-\mathrm{n}$ based AC-OLEDs. We further compare our findings with theoretical predictions which are based on the sample geometry, material constants, and the assumption of Zener tunneling as the most probable mechanism for charge regeneration. We observe a reduction in driving voltage with increasing insulator capacitance as well as with decreasing thickness of the intrinsic organic layer. Our findings are in good agreement with theoretically calculated values based on a three-capacitor device model. The obtained onset field strengths for light emission are close to the predicted breakdown field strength for Zener tunneling in the wide gap organic materials used in this study.

A series of devices with the p-i-n device architecture illustrated in Fig. 1 was fabricated. All layers were deposited in a UHV chamber at a base pressure of about $10^{-8}$ mbar onto glass substrates coated with structured tin-doped indium oxide (ITO) which acts as transparent bottom electrode. Doping was realized by co-evaporation of the matrix material and the dopant. The $\mathrm{HfO}_{2}$ insulating layers were deposited by radio frequency (RF) magnetron sputtering in a high 
purity argon atmosphere without any reactive gas at a pressure of $5 \cdot 10^{-3}$ mbar. A rate of $2 \AA / \mathrm{s}$ was achieved for an RF power of $100 \mathrm{~W}$. For the second insulating layer located on top of the organic material, the power was reduced to $60 \%-70 \%$ of its initial value to prevent sputter-induced damage of the underlying organic material. $\mathrm{HfO}_{2}$ was chosen as insulating material because of its high dielectric constant and therefore strong polarizability, as well as the high band-gap of $5.8 \mathrm{eV}$ which provides sufficiently high injection barriers for both holes and electrons. ${ }^{15}$

For the p-type and n-type transport layers, we used $\mathrm{N}, \mathrm{N}, \mathrm{N}^{\prime}, \mathrm{N}^{\prime}$-tetrakis(4-methoxyphenyl)-benzidine (MeO-TPD) doped with 6 wt. \% 2,2'-(perfluoronaphthalene-2,6-diylidene) dimalononitrile (F6-TCNNQ) and 4,7-diphenyl-1,10phenanthroline (BPhen) doped with cesium (Cs), respectively. An electron and a hole blocking layer (EBL/HBL) were used to confine the charge carriers and excitons inside the emission layer (EML). The HBL was realized using a layer of BPhen, whereas 2,2',7,7'-tetrakis-(N,N-diphenylamino)-9, $9^{\prime}$-spirobifluorene (Spiro-TAD) was used for the EBL. The EML consists of N, $\mathrm{N}^{\prime}$-di(naphthalen-1-yl)-N-N'diphenyl-benzidine $(\alpha-\mathrm{NPD})$ doped with $10 \mathrm{wt} \%$ of the highly efficient phosphorescent red emitter Iridium(III)bis (2-methyldibenzo-[f,h]chinoxalin)(acetylacetonat) (Ir(MDQ) (acac)). A $100 \mathrm{~nm}$ thick layer of aluminum was used as highly reflective top electrode. The active area $A$ of the device is determined by the overlap of bottom and top electrode, which was $5.96 \mathrm{~mm}^{2}$. Prior to device investigation, the samples were encapsulated in a nitrogen glovebox.

The thickness of the intrinsic EBL and HBL is denoted with $x$, and the thickness of the EML is denoted with $y$. We tested four devices with $x / y=10 \mathrm{~nm} / 20 \mathrm{~nm}, 10 \mathrm{~nm} / 15 \mathrm{~nm}$, $10 \mathrm{~nm} / 10 \mathrm{~nm}$, and $7.5 \mathrm{~nm} / 10 \mathrm{~nm}$, respectively. The thickness of the $\mathrm{HfO}_{2}$ insulating layer was $120 \mathrm{~nm}$ for these four devices (Fig. 1(a)). In another series of devices, the thickness of the $\mathrm{HfO}_{2}$ layer was varied using the intrinsic layer configuration with $x / y=7.5 \mathrm{~nm} / 10 \mathrm{~nm}$. Here, we varied the oxide thickness from $z=120 \mathrm{~nm}$ to $180 \mathrm{~nm}$ in $20 \mathrm{~nm}$ steps (Fig. 1(b)).

Optical simulations based on a radiative dipole model were used to model the light-extraction efficiency and to ensure that the outcoupling efficiency is comparable in all devices. ${ }^{14}$ This was achieved by adjusting the HTL and ETL thickness $\left(\mathrm{d}_{H T L}, \mathrm{~d}_{E T L}\right)$ such that the recombination zone was located in the maximum of the optical field in all devices. $\mathrm{d}_{H T L}$ was varied between $74 \mathrm{~nm}$ and $88 \mathrm{~nm}$ and $\mathrm{d}_{\text {ETL }}$ ranged from $50 \mathrm{~nm}$ to $108 \mathrm{~nm}$. Due to the use of doped HTLs and ETLs, the variation in transport layer thickness was not expected to lead to significant changes in electrical device characteristics.

The electrical driving of the samples, the measurement setup, and the correct determination of the luminous efficacy for this kind of devices have been described in previous publications. ${ }^{8,12}$ All measurements were performed at a fixed frequency of $5 \mathrm{kHz}$. Voltages are given as RMS (root-meansquare) values if not stated otherwise.

In previous studies on p-i-n AC-OLEDs, we assumed Zener tunneling of electrons from the HOMO of the p-type layer to the LUMO of the n-type layer to be responsible for refilling the depleted transport layers and to provide charge carriers for light emission. In low gap organic tunnel diodes, Zener breakdown occurs at field strengths of 1 . $1.5 \mathrm{MV} / \mathrm{cm}^{13,16}$ In the wide gap materials used in this study, a breakdown field strength of around $3 \mathrm{MV} / \mathrm{cm}$ for Zener tunneling is predicted, assuming a triangular tunneling barrier where the relation between electric breakdown field $F_{b}$ and energy gap $E_{g}$ is given by $F_{b} \sim E_{g}^{3 / 2}$ (Fowler-Nordheim). ${ }^{17}$

The AC-OLED can be modeled electrically as a series of three capacitors with capacitors 1 and 3 representing the insulating layers and capacitor 2 being the intrinsic organic material, that is, EBL, EML, and HBL. For capacitors connected in series, the transferred charge $Q$ is a constant given by

$$
Q=C_{1} \times \Delta V_{1}=C_{2} \times \Delta V_{2}=\ldots=\text { const. },
$$

where $C$ is the capacitance and $\Delta V$ the voltage drop across the corresponding capacitor. The electrodes as well as HTL and ETL of our devices are highly conductive; thus, the voltage drop across these layers can be neglected. The driving voltage $V_{d}$ is therefore the sum of the drop in voltage across the three capacitors in the device

$$
V_{d}=2 \times \Delta V_{\text {ox }}+\Delta V_{\text {org }} .
$$

\begin{tabular}{|lr|}
\hline aluminum & $100 \mathrm{~nm}$ \\
\hline hafnium dioxide & $120 \mathrm{~nm}$ \\
\hline BPhen:Cs & $\mathrm{d}_{\mathrm{ETL}}$ \\
\hline BPhen & $\mathrm{x} \mathrm{nm}$ \\
\hline a-NPD:Ir(MDQ $)_{2}(\mathrm{acac})(10 \mathrm{wt} \%)$ & $\mathrm{y} \mathrm{nm}$ \\
\hline Spiro-TAD & $\mathrm{x} \mathrm{nm}$ \\
\hline MeO-TPD:F6-TCNNQ (6wt $\%)$ & $\mathrm{d}_{\mathrm{HTL}}$ \\
\hline hafnium dioxide & $120 \mathrm{~nm}$ \\
\hline \multicolumn{2}{|c}{ glass substrate } \\
\hline \multicolumn{2}{|c}{$90 \mathrm{~nm}$} \\
\hline
\end{tabular}

(a)

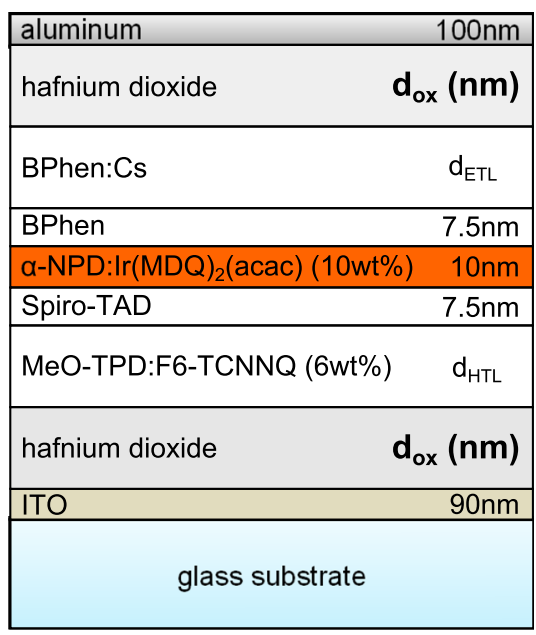

FIG. 1. Schematic illustration of the stack architecture of the investigated devices for (a) the thickness variation of the intrinsic organic layers and (b) the variation of the oxide thickness, respectively. For all devices, the recombination zone is shifted to the optical field maximum by adjusting the HTL and ETL thickness $\left(\mathrm{d}_{H T L}\right.$, $\left.\mathrm{d}_{\text {ETL }}\right)$. 
Using Eqs. (1) and (2), $\Delta V_{o x}$ and $\Delta V_{\text {org }}$ can be calculated. Division by the respective layer thickness yields the electric field across the insulating oxide layers $F_{o x}$ and the intrinsic organic layers $F_{\text {org }}$ according to

$$
\begin{gathered}
F_{o r g}=\frac{V_{d}}{d_{o r g} \times\left(2 \times \frac{C_{o r g}}{C_{o x}}+1\right)}, \\
F_{o x}=\frac{V_{d}}{d_{o x} \times\left(\frac{C_{o x}}{C_{o r g}}+2\right)}=\frac{\epsilon_{o r g}}{\epsilon_{o x}} \times F_{o r g} .
\end{gathered}
$$

The capacitance of the oxide layers as well as the intrinsic organic layers can be calculated from the active area A and the corresponding dielectric constant of each material which is $\epsilon_{o x}=23$ in case of $\mathrm{HfO}_{2}$ and $\epsilon_{\text {org }} \approx 2.8$ in case of the organic material. Using these values and Eq. (3), the driving voltage required to reach a certain field strength across the intrinsic organic layers can be estimated.

Figure 2(a) shows the driving voltage $V_{d}$ required for four different field strengths as a function of the different intrinsic organic layer thicknesses used in this study. These calculations show that reducing the intrinsic organic layer thickness by $5 \mathrm{~nm}$ results in reduction in driving voltage of around $1.5 \mathrm{~V}$ and $2.7 \mathrm{~V}$ for field strengths of $3.0 \mathrm{MV} / \mathrm{cm}$ and $5.4 \mathrm{MV} / \mathrm{cm}$, respectively. Similar results are obtained when varying the thickness of the insulating oxide layer and comparable reductions in driving voltage are expected (Fig. 2(b)).

The field strength in the oxide layers and the intrinsic organic layers are linearly correlated through the ratio of the dielectric constants of these layers (Eq. (4)). Thus, even for very high $F_{\text {org }}$ values of $5.4 \mathrm{MV} / \mathrm{cm}$, the field across insulating $\mathrm{HfO}_{2}$ layers is only $0.66 \mathrm{MV} / \mathrm{cm}$ and therefore lower than the breakdown field strength of $\mathrm{HfO}_{2}$ which is in the range of $1-2 \mathrm{MV} / \mathrm{cm}$. Charge carrier injection can therefore be ruled out as origin of exciton formation in our devices as we have already shown in previous publications. ${ }^{9,10,12}$

The experimental results of our first series of devices with different thickness of the intrinsic organic layer (Fig. 1(a)) are summarized in Fig. 3. While the general shape of the luminance-voltage curves remains identical for all thicknesses, the voltage required to achieve a certain brightness can be considerably reduced when reducing the thickness of the intrinsic organic layers (Fig. 3(a)). A $5 \mathrm{~nm}$ reduction in intrinsic organic layer thickness leads to a decrease in driving voltage by approximately $1.5-2 \mathrm{~V}$ which is in good agreement with the values in Fig. 2(a). We can identify two distinct regions in each luminance curve. The first region (approximately $1-10 \mathrm{~cd} / \mathrm{m}^{2}$ ), for which the luminance is only slowly increasing with voltage, is mainly governed by small residual leakage currents and not tunneling. This parasitic leakage current regime is also present in low-gap organic tunnel diodes. ${ }^{13,16}$ The second region $\left(>10 \mathrm{~cd} / \mathrm{m}^{2}\right)$, for which the slope of the luminance curve is greatly increased, can be ascribed to the Zener tunneling regime where tunneling currents are the major contribution to charge regeneration. The transition from the first to the second is defined as the onset of tunneling. Interestingly, the field strength at the onset is close to $3.05 \mathrm{MV} / \mathrm{cm}$ for all four samples (Fig. 3(b)). This value is in good agreement with theory according to which the transition to the tunneling regime is supposed to occur at around $3 \mathrm{MV} / \mathrm{cm}$.

When varying the thickness of the $\mathrm{HfO}_{2}$ oxide layer (Fig. 4), we observe an almost identical behavior as for the variation of the intrinsic organic layers (Fig. 3). Most importantly, the onset field strengths are again very similar for devices with different oxide layer thickness and again agree with the expected value of $\approx 3 \mathrm{MV} / \mathrm{cm}$ (Fig. 4(b)).

Additionally, Figs. 3(b) and 4(b) also show the estimated field strength across the intrinsic organic layers required for a device luminance of $100 \mathrm{~cd} / \mathrm{m}^{2}$ and $1000 \mathrm{~cd} / \mathrm{m}^{2}$. As for the onset of luminance, the field strength is nearly identical for all intrinsic and insulating layer thicknesses investigated here. The field required for a luminance of $100 \mathrm{~cd} / \mathrm{m}^{2}$ and $1000 \mathrm{~cd} / \mathrm{m}^{2}$ is $3.8-3.9 \mathrm{MV} / \mathrm{cm}$ and $5.5-5.6$ $\mathrm{MV} / \mathrm{cm}$, respectively.

Table I summarizes the expected impedance of all samples in this study. These values were calculated for a frequency of $5 \mathrm{kHz}$ using only the sample geometry and the dielectric constants of the involved materials. Similar values were obtained for the variation in intrinsic layer thickness and for the variation in oxide thickness.

The experimentally obtained impedance values for the variation of the intrinsic organic layers and the insulating layers are shown in Figs. 3(c) and 4(c), respectively. The low voltage impedance is consistent with the theoretically predicted value for the respective layer thicknesses for all samples in this study. The tunneling onset can be clearly distinguished in Figs. 3(c) and 4(c) as a sudden drop in
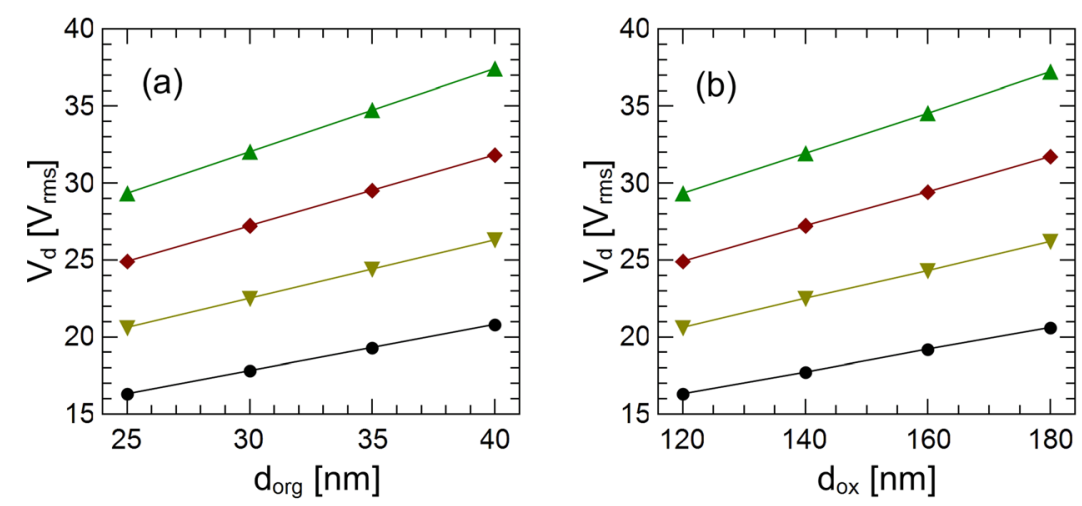

FIG. 2. Calculated driving voltage $V_{d}$ required to obtain electric fields of 3 , $3.8,4.6$, and $5.4 \mathrm{MV} / \mathrm{cm}$ for several thicknesses of (a) the intrinsic organic layers and (b) the insulating oxide layers. In case of (a), the oxide thickness is kept constant at $120 \mathrm{~nm}$, whereas the intrinsic organic layer has a constant thickness of $25 \mathrm{~nm}$ in case of (b).

field strength: $\multimap 3 \mathrm{MV} / \mathrm{cm} \longrightarrow 3.8 \mathrm{MV} / \mathrm{cm} \longrightarrow 4.6 \mathrm{MV} / \mathrm{cm} \longrightarrow 5.4 \mathrm{MV} / \mathrm{cm}$ 

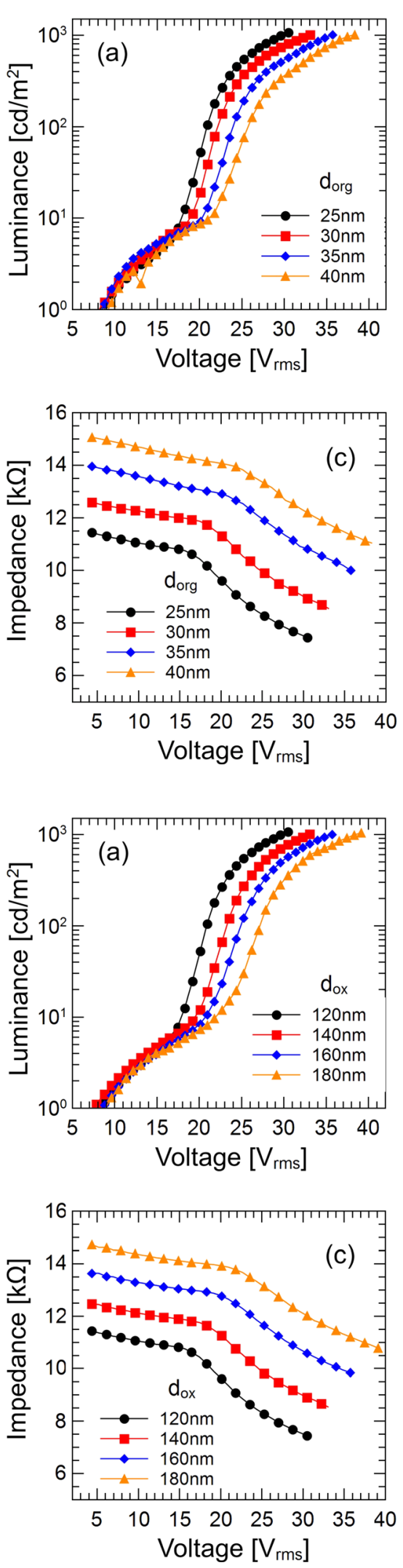
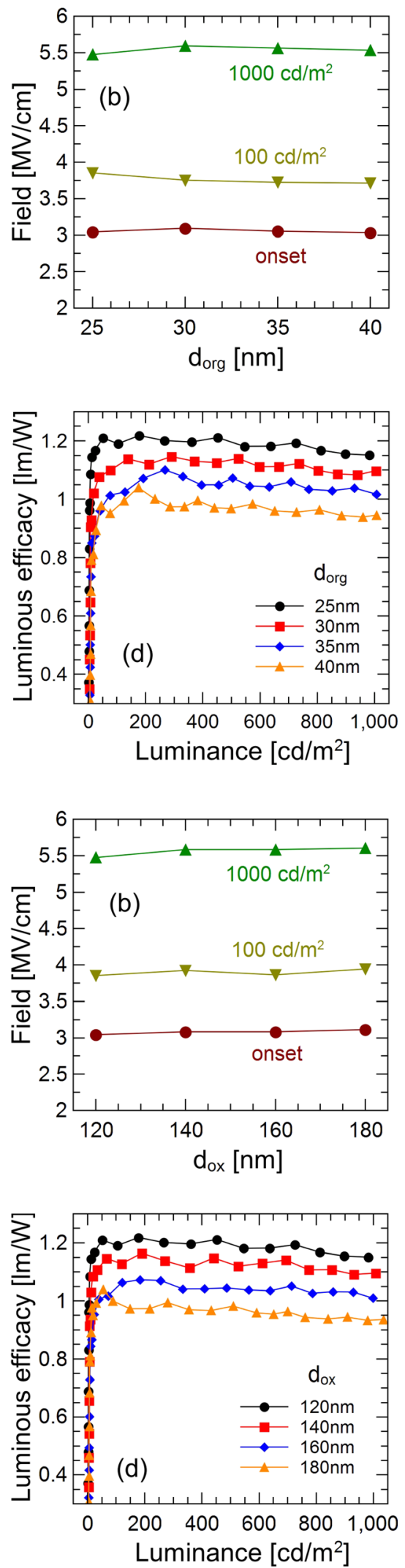

FIG. 3. Electrical characteristics of a series of AC-OLEDs with different thicknesses of the intrinsic organic layers, that is, EBL, EML, and HBL, and constant thicknesses of the $\mathrm{HfO}_{2}$ oxide layers of $120 \mathrm{~nm}$. (a) Voltageluminance characteristics and (b) field strength derived from these characteristics to reach the onset of light emission, as well as luminance levels of $100 \mathrm{~cd} / \mathrm{m}^{2}$ and $1000 \mathrm{~cd} / \mathrm{m}^{2}$, shown as a function of the intrinsic organic layer thickness. (c) Voltage-impedance characteristics and (d) luminous efficacy for the studied devices.
FIG. 4. Electrical characteristics of a series of AC-OLEDs with different thicknesses of the insulating $\mathrm{HfO}_{2}$ oxide layers and a constant intrinsic layer thickness of $25 \mathrm{~nm}$. (a) Voltageluminance characteristics and (b) field strength derived from these characteristics to reach the light emission onset, $100 \mathrm{~cd} / \mathrm{m}^{2}$ and $1000 \mathrm{~cd} / \mathrm{m}^{2}$, shown as a function of the insulator thickness. (c) Voltage-impedance characteristics and (d) luminous efficacy for the different devices. impedance at a voltage which coincides with the onset voltage derived from the luminance curves. At this point, the intrinsic organic layers lose their capacitive nature due to injection into this formerly depleted region. The overall device capacitance, which is now only determined by both insulating layers, increases, and the measured impedance is reduced accordingly.
Figures 3(d) and 4(d) show the luminous efficacy as a function of the luminance. As a result of the reduced driving voltage, the luminous efficacy is increased by up to $20 \%$ for both types of variation.

In summary, the influence of the insulator thickness and the intrinsic organic layer thickness on the driving voltage of p-i-n based AC-OLEDs was investigated. A three-capacitor 
TABLE I. Calculated device impedance $\mathrm{Z}$ for a frequency of $5 \mathrm{kHz}$.

\begin{tabular}{|c|c|c|c|c|c|c|c|c|}
\hline \multirow[b]{2}{*}{$\mathrm{d}_{\text {org } / \text { ox }}(\mathrm{nm})$} & \multicolumn{4}{|c|}{ Intrinsic variation } & \multicolumn{4}{|c|}{ Oxide variation } \\
\hline & 40 & 35 & 30 & 25 & 180 & 160 & 140 & 120 \\
\hline $\mathrm{Z}(\mathrm{k} \Omega)$ & 14.9 & 13.8 & 12.8 & 11.7 & 14.8 & 13.8 & 12.7 & 11.7 \\
\hline
\end{tabular}

model was employed for basic device behavior predictions and good agreement with the experimental values was obtained. For all samples, the measured device impedance was close to the calculated value which indicates that the thickness of all layers was controlled precisely and that no inductive or parasitic, Ohmic loss channels are present. Furthermore, we showed that a significant increase in light emission occurs at field strengths of 3-3.1 MV/cm across the intrinsic organic layers, in agreement with Fowler-Nordheim theory predicting, a minimum field strength of $3 \mathrm{MV} / \mathrm{cm}$ required for Zener tunneling in wide band gap organic materials. In terms of device improvement, we were able to reduce the driving voltage by up to $23 \%$ which results in a luminous efficacy enhancement of $20 \%$. Reducing the insulating layer thickness below $120 \mathrm{~nm}$ can lead to further improvements in driving voltage. However, thin oxide layers are prone to increased leakage currents which result in lowering the luminous efficacy of the device. A further reduction of the intrinsic organic layers is also limited, as EML and blocking layers require a certain minimum thickness for efficient exciton generation and exciton blocking.

This work was funded with financial means of the European Social Fund and the Free State of Saxony through the OrthoPhoto project.

${ }^{1}$ G. Destriau, J. Chim. Phys. 33, 587 (1936).

${ }^{2}$ P. F. Smet, I. Moreels, Z. Hens, and D. Poelman, Materials 3, 2834 (2010).

${ }^{3}$ D. C. Krupka, J. Appl. Phys. 43, 476 (1972).

${ }^{4}$ Y. Z. Wang, D. D. Gebler, L. B. Lin, J. W. Blatchford, S. W. Jessen, H. L. Wang, and A. J. Epstein, Appl. Phys. Lett. 68, 894 (1996).

${ }^{5}$ T. Tsutsui, S.-B. Lee, and K. Fujita, Appl. Phys. Lett. 85, 2382 (2004).

${ }^{6}$ J. Sung, Y. S. Choi, S. J. Kang, S. H. Cho, T.-W. Lee, and C. Park, Nano Lett. 11, 966 (2011).

${ }^{7}$ Y. Chen, Y. Xia, G. M. Smith, Y. Gu, C. Yang, and D. L. Carroll, Appl. Phys. Lett. 102, 013307 (2013).

${ }^{8}$ M. Fröbel, A. Perumal, T. Schwab, C. Fuchs, K. Leo, and M. C. Gather, Phys. Status Solidi A 210, 2439 (2013).

${ }^{9}$ A. Perumal, M. Fröbel, S. Gorantla, T. Gemming, B. Lüssem, J. Eckert, and K. Leo, Adv. Funct. Mater. 22, 210 (2012).

${ }^{10}$ A. Perumal, B. Lüssem, and K. Leo, Appl. Phys. Lett. 100, 103307 (2012).

${ }^{11}$ A. Perumal, B. Lüssem, and K. Leo, Org. Electron. 13, 1589 (2012).

${ }^{12}$ M. Fröbel, A. Perumal, T. Schwab, M. C. Gather, B. Lüssem, and K. Leo, Org. Electron. 14, 809 (2013).

${ }^{13}$ H. Kleemann, R. Gutierrez, F. Lindner, S. Avdoshenko, P. D. Manrique, B. Lüssem, G. Cuniberti, and K. Leo, Nano Lett. 10, 4929 (2010).

${ }^{14}$ M. Furno, R. Meerheim, S. Hofmann, B. Lüssem, and K. Leo, Phys. Rev. B 85, 115205 (2012).

${ }^{15}$ J. Robertson, Eur. Phys. J. Appl. Phys. 28, 265 (2004).

${ }^{16}$ H. Kleemann, K. Leo, B. Lüssem, R. Gutierrez, S. Avdoshenko, and G. Cuniberti, Org. Electron. 14, 193 (2013).

${ }^{17}$ R. H. Fowler and L. Nordheim, Proc. R. Soc. London, Ser. A 119, 173 (1928). 